\title{
Strong dynamical screening of the $(e, 2 e)$ processes for electron impact ionization of helium in the perpendicular plane symmetric geometry
}

\author{
Zhan-Bin Chen* \\ College of Physics and Electrical Information, Northwest Normal University, Lanzhou \\ 730070, China
}

Received 1 July 2011; Accepted (in revised version) 3 August 2011

Published Online 18 February 2012

\begin{abstract}
We apply BBK, DS3C model and the new modified Sommerfeld parameters of the present DS3C model to calculation of the triple differential cross sections (TDCS) for electron impact ionization of helium in the perpendicular plane symmetric geometry. The results of the present are compared with the measurements and those of other theoretical models. It was found that the present results give a better description for the experimental data and the dynamical screening effects are strong in this geometry. The influence of the Sommerfeld parameters on the triple differential cross sections is also analyzed and discussed in the perpendicular plane geometry.
\end{abstract}

PACS: 34.80.Dp, 34.50.Fa

Key words: perpendicular plane geometry, doubly-symmetric, sommerfeld parameter

\section{Introduction}

Electron-impact atom ionization provides a very interesting diversity of phenomena because of the wide range of kinematical situations available to the three-body final state. The incident electron knocks out the target electron with the remainder of the target atom acting as an inert core. An $(e, 2 e)[1-8]$ reaction is the measurement of the electron-impact ionization process where both the existing electrons are detected in coincidence. It is a measurement almost at the limit of what is quantum mechanically known and its description presents a substantial challenge to theory. Over the past 30 years, significant progress has been made on the theoretical side, with several theories demonstrating excellent agreement with a variety of experimental data yielding multiple differential cross sections for He [9-12], for a variety of outgoing electron geometries and kinematics, and from near threshold to relatively high

\footnotetext{
${ }^{*}$ Corresponding author. Email address: 253072513@qq.com (Z. B. Chen)
} 
impact energies in the coplanar geometry. But in the non-coplanar geometry constitute a major challenge for theoretical models for many years.

A significant advance in the theory of electron impact ionization has been the use of the asymptotically correct Coulomb three-body wavefunction for ejected and scattered electrons in the field of the residual ion by Brauner, Briggs and Klar(BBK), The BBK model [1] is valid for both symmetric and asymmetric geometries at intermediate energy. Unfortunately, the BBK model is not in agreement with the measurements at low energies because the normalization factor corresponding to the repulsive e-e interaction goes exponentially to zero as the total energy above threshold of the two electrons goes to zero. This exponential decrease also causes the magnitude of the cross section to decrease exponentially. So, Berakdar [13] corrected the deficiency of the BBK wavefunction, while still maintaining the philosophy behind it, by the introduction of effective Sommerfeld parameters in the two-body factors in the BBK wavefunction, and the results turn out to be in good agreement with experimental findings over a wide range of both the coplanar and the non-coplanar geometry.

In our earlier paper [4], we calculated the TDCS for electron impact ionization of helium in a symmetric non-coplanar energy-sharing geometry at incident energies from 27.6 $\mathrm{eV}$ to $84.6 \mathrm{eV}$ using the DS3C model presented by Berakdar found the results not excellent agreement with the measurement $[14,15]$. Berakdar has successfully derived an approximate analytical solution of the quantum-mechanical three body Coulomb continuum problem. However, his work is not very perfectly in the non-coplanar geometry or perpendicular plane geometry.

Hence, in this paper, we apply BBK, DS3C model and the new modified Sommerfeld parameters of the DS3C model to calculation of TDCS for electron impact ionization of helium in the perpendicular plane symmetric geometry.The results of the present are compared with the absolute measurements and those of other theoretical models. The influence of the Sommerfeld parameters on the triple differential cross sections is also analyzed and discussed in the perpendicular plane.

\section{Theory}

In atomic units, the TDCS for the $(e, 2 e)$ process, is given by

$$
\operatorname{TDCS}=(2 \pi)^{4} \frac{k_{1} k_{2}}{k_{0}}\left|\frac{3}{4}\right| f\left(\vec{k}_{1}, \vec{k}_{2}\right)-\left.f\left(\vec{k}_{2}, \vec{k}_{1}\right)\right|^{2}+\frac{1}{4}\left|f\left(\vec{k}_{1}, \vec{k}_{2}\right)+f\left(\vec{k}_{2}, \vec{k}_{1}\right)\right|^{2} \mid,
$$

where $k_{0}, k_{1}$ and $k_{2}$ are the momenta of the incident, the scattered and the ejected electrons, respectively. The direct amplitude reads

$$
f\left(k_{1}, k_{2}\right)=\left\langle\psi_{f}^{-}\left|V_{i}\right| \phi_{i}\right\rangle
$$

with

$$
V_{i}=-\frac{2}{r_{1}}+\frac{1}{\left|\vec{r}_{1}-\vec{r}_{2}\right|}+\frac{1}{\left|\vec{r}_{1}-\vec{r}_{3}\right|}
$$


where $r_{1}$ is the projectile position, $r_{2}$ and $r_{3}$ are the target electron positions. $\psi_{f}^{-}$and $\psi_{i}$ represent the wavefunctions describing the whole system in its final and initial states, respectively.

The initial state $\psi_{i}$ is a product of an incoming plane wave representing the impinging projectile with momentum $k_{0}$ and an undistorted 1s-state of atomic helium, and can be represented by the asymptotic wavefunction

$$
\psi_{i}\left(\vec{r}_{0}, \vec{r}_{1}, \vec{r}_{2}, \vec{r}_{3}\right)=(2 \pi)^{-\frac{3}{2}} e^{i \vec{k}_{0} \cdot \vec{r}_{1}} \varphi\left(\vec{r}_{2}, \vec{r}_{3}\right)
$$

with

$$
\varphi\left(\vec{r}_{2}, \vec{r}_{3}\right)=\varphi\left(\vec{r}_{2}\right) \varphi\left(\vec{r}_{3}\right)
$$

where $\varphi(\vec{r})$ is the product of the two-exponential-function fit to the Hartree-Fock wavefunctions given by

$$
\varphi(\vec{r})=\sqrt{\frac{1}{4 \pi}}\left(g_{a} e^{-\lambda_{a} r}+g_{b} e^{-\lambda_{b} r}\right),
$$

where $\lambda_{a}=1.41, \lambda_{b}=2.61, g_{a}=2.60505$ and $g_{b}=2.08114$. This helium ground state contains some radial correlation but no angular correlation.

The final-state wavefunction $\psi_{f}^{-}$given by

$$
\psi_{f}^{-}\left(\vec{r}_{1}, \vec{r}_{2}, \vec{r}_{3}\right)=\phi_{f}\left(\vec{r}_{1}, \vec{r}_{2}\right) \phi_{\text {ion }}\left(\vec{r}_{3}\right),
$$

where $\phi_{\text {ion }}\left(\vec{r}_{3}\right)$ is the wavefunction of the hydrogenic ion $\mathrm{He}^{+}$, which can be given by

$$
\phi_{\text {ion }}\left(\vec{r}_{3}\right)=\sqrt{\frac{8}{\pi}} e^{-2 r_{3}}
$$

And $\psi_{f}^{-}$will be described by the BBK wavefunction in its general form

$$
\psi_{f}^{-}=M e^{i \vec{k}_{1} \cdot \vec{r}_{1}} e^{i \vec{k}_{2} \cdot \vec{r}_{2}} \chi\left(\vec{r}_{1}, \vec{r}_{2}\right)
$$

with

$$
\begin{aligned}
& M=\frac{1}{(2 \pi)^{3}} \exp \left[-\frac{\pi}{2}\left(\alpha_{1}+\alpha_{2}+\alpha_{12}\right)\right] \Gamma\left(1-i \alpha_{1}\right) \Gamma\left(1-i \alpha_{2}\right) \Gamma\left(1-i \alpha_{12}\right) \\
& \chi\left(\vec{r}_{1}, \vec{r}_{2}\right)=\prod_{j=1}^{2}{ }_{1} F_{1}\left[i \alpha_{j} ; 1:-i\left(k_{j} r_{j}+\vec{k}_{j} \cdot \vec{r}_{j}\right)\right]_{1} F_{1}\left[i \alpha_{12} ; 1 ;-i\left(k_{12} r_{12}+\vec{k}_{12} \cdot \vec{r}_{12}\right)\right],
\end{aligned}
$$

where $\alpha$ is called the Sommerfeld parameter can be given by

$$
\alpha_{1}=-\frac{Z}{k_{1}}, \quad \alpha_{2}=-\frac{Z}{k_{2}}, \quad \alpha_{12}=\frac{1}{2 k_{12}}
$$


with

$$
\vec{k}_{12}=\frac{1}{2}\left(\vec{k}_{1}-\vec{k}_{2}\right)
$$

And $Z$ is the charge of the residual ion. Equation (Eq. 9) is called the $3 C$ wavefunction. So this model is called BBK model [1].

As can be seen in Ref. [13], by considered a dynamic screening (DS) of the three two body Coulomb interactions, the ground state of He is described by the Sommerfeld parameters

$$
\begin{aligned}
& \beta_{1}=\beta_{2}=-\frac{4 Z-\sin \xi}{4 k} \\
& \beta_{12}=\frac{1-\sin ^{2} \xi}{2 k \sin \xi}
\end{aligned}
$$

with

$$
\xi=\frac{\left(\cos ^{-1} \vec{k}_{1} \cdot \vec{k}_{2}\right)}{2}
$$

where $k_{1}=k_{2}$ and $\xi=\left(\cos ^{-1} \vec{k}_{1} \cdot \vec{k}_{2}\right) / 2$ is the angle between $\vec{k}_{1}$ and $\vec{k}_{2} . Z$ is the effective charge of $\mathrm{He}^{+}(1 \mathrm{~s})$ core seen by the escaping electrons. This model is called DS3C model in the doubly-symmetric geometry.

In the perpendicular plane geometry, the outgoing channel distortions and short and long range correlations between all electrons and the core and the effective shield of residual electron in the final state of $\mathrm{He}^{+}$(ls) core are important. By considered those, the new modified Sommerfeld parameters is readily established that these conditions are satisfied by

$$
\begin{aligned}
& \gamma_{i}=-\frac{\left(4 Z_{i}-\sin \xi\right)}{4 k_{i}} \quad(i=1,2) \\
& \gamma_{12}=-\gamma_{1}-\gamma_{2}+\alpha_{1}+\alpha_{2}+\alpha_{12}
\end{aligned}
$$

with

$$
Z_{i}=1-\frac{2}{\left(1+4 k_{i}^{2} \sin ^{2}\left(\frac{\xi}{2}\right)\right)^{2}} \quad(i=1,2),
$$

where $\xi=\left(\cos ^{-1} \vec{k}_{1} \cdot \vec{k}_{2}\right) / 2$ is the angle between $\vec{k}_{1}$ and $\vec{k}_{2}$.

In the non-coplanar geometry, the scattering angles $\xi_{1}$ and $\xi_{2}$ and the gun angle $\psi$ is related to the scattering angles $\theta_{1}$ and $\theta_{2}$ and the relative azimuthal angle $\phi$ considered in the theory, by the following relations

$$
\begin{aligned}
& \cos \theta=\cos \xi \cos \psi, \\
& \cot \frac{\phi}{2}=\cot \xi \sin \psi .
\end{aligned}
$$

For perpendicular plane symmetric geometry (Fig. 1), $\xi_{1}=\xi_{2}=\xi$ and $\psi=90^{\circ}$. 


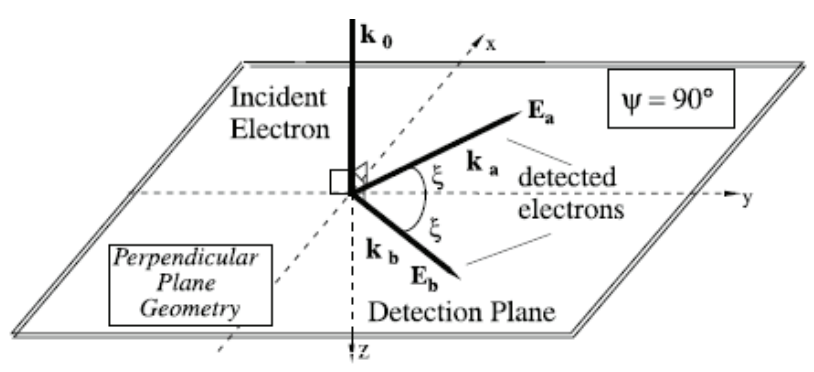

Figure 1: The perpendicular plane symmetric geometry.

\section{Discussion}

We have calculated the TDCS for electron impact ionization of helium using the BBK, DS3C model and the new modified Sommerfeld parameters of the present DS3C model in the perpendicular plane symmetric geometry at incident energies of 64.6, 74.6, 84.6, and $104.6 \mathrm{eV}$. The results are given in Fig. 2(a)-(h), compared with the experimental data in Ref $[15,16]$. The calculated results are normalized for the best visual agreement with the experiment.

It can be seen from Fig. 2(a)-(d), the three peaks at low energies evolves into a two peak structure as the energy increases. At incident energy of $74.6 \mathrm{eV}$ the three peaks are of approximately the same magnitude. And the relative magnitude of the central peak at $\xi=\pi / 2$ compared to the peaks at low with increasing energy. In Fig. 2(a)-(d) at incident energy of 64.6 to $104.6 \mathrm{eV}$, the DS3C model is in good agreement with experiment in $\xi=$ $45^{\circ}$ and $\xi=135^{\circ}$ peaks, though small quantitative discrepancies remain. The BBK model underestimate of cross sections in $\xi=45^{\circ}$ and $\xi=135^{\circ}$ peaks are corrected by DS3C model. More specifically, the DS3C still agreement with angular distribution of the cross sections, not the BBK model especially for incident energy $74.6 \mathrm{eV} 84.6 \mathrm{eV}$ (Fig. 2(b)(c)). While, the results of the DS3C model and the BBK calculations are not agreement with the experiment in $\xi=90^{\circ}$ peak in Fig. 2. Our present DS3C approach is in full agreement with any experiment results, especially in the $\xi=90^{\circ}$ peak, the experiment shows a high peak in this region that is improved by our present DS3C to some extent, not the BBK and DS3C calculations.

In Fig. 2(f)-(h), TDCS results in the perpendicular plane symmetric unequal-energy-sharing geometry are shown. In this case, same as Fig. 2(a)-(d), our present DS3C and experiment now agree in the any shape of the cross section, especially in the $\xi=90^{\circ}$ peak. It is also not the BBK and DS3C calculations.

Moreover, it is very encouraging to see that the DS3C calculations show the improvement over the BBK results are in excellent agreement with the experimental measurements at Fig. 2(a)(b)(c)(h). However, the results of the BBK model are more agreement with the experiment than DS3C model in the $\xi=90^{\circ}$ peak at Fig. 2(d)(f).

To sum up, our present DS3C model shows great improvement over the BBK and DS3C 
model in obtaining better magnitudes and shapes of cross sections compared with Experiment in the perpendicular plane symmetric equal-energy-sharing and unequal-energy-sharing geometry, including the angular positions and relative heights. It gives a better description for the experimental data. Strong differences among our present DS3C to DS3C or 3C resulting from residual electron in the final state of $\mathrm{He}^{+}$core dynamic screening (DS) can be observed. It shows in this geometry the outgoing channel distortions and short and long range correlations between all electrons and the core must be included. It also shows that our new Sommerfeld parameters to description these effects are rather effectively and perfectly.

In order to description these effects and the effective shield of residual electron in the final state of $\mathrm{He}^{+}$core in the perpendicular plane symmetric geometry, we give the effective charge $Z$ plotted as a function of the ejection angle $\xi$ of Fig. 2(a)

It can be seen from Fig. 2(e), effective charges $Z$ at $\xi=90^{\circ}$ is 0.75 in DS3C model but 0.712 in our present DS3C, differences between the present DS3C and DS3C of the effective charges $Z$ caused different cross-sections in Fig. 2 especially in the $\xi=90^{\circ}$ peak. Moreover, the effective charges $Z$ and new Sommerfeld parameters description the modification of the strength of a particular two-body Coulomb interaction depends on the momenta of the two particles relative to the third one, which represents a dynamic screening (DS) of the three two-
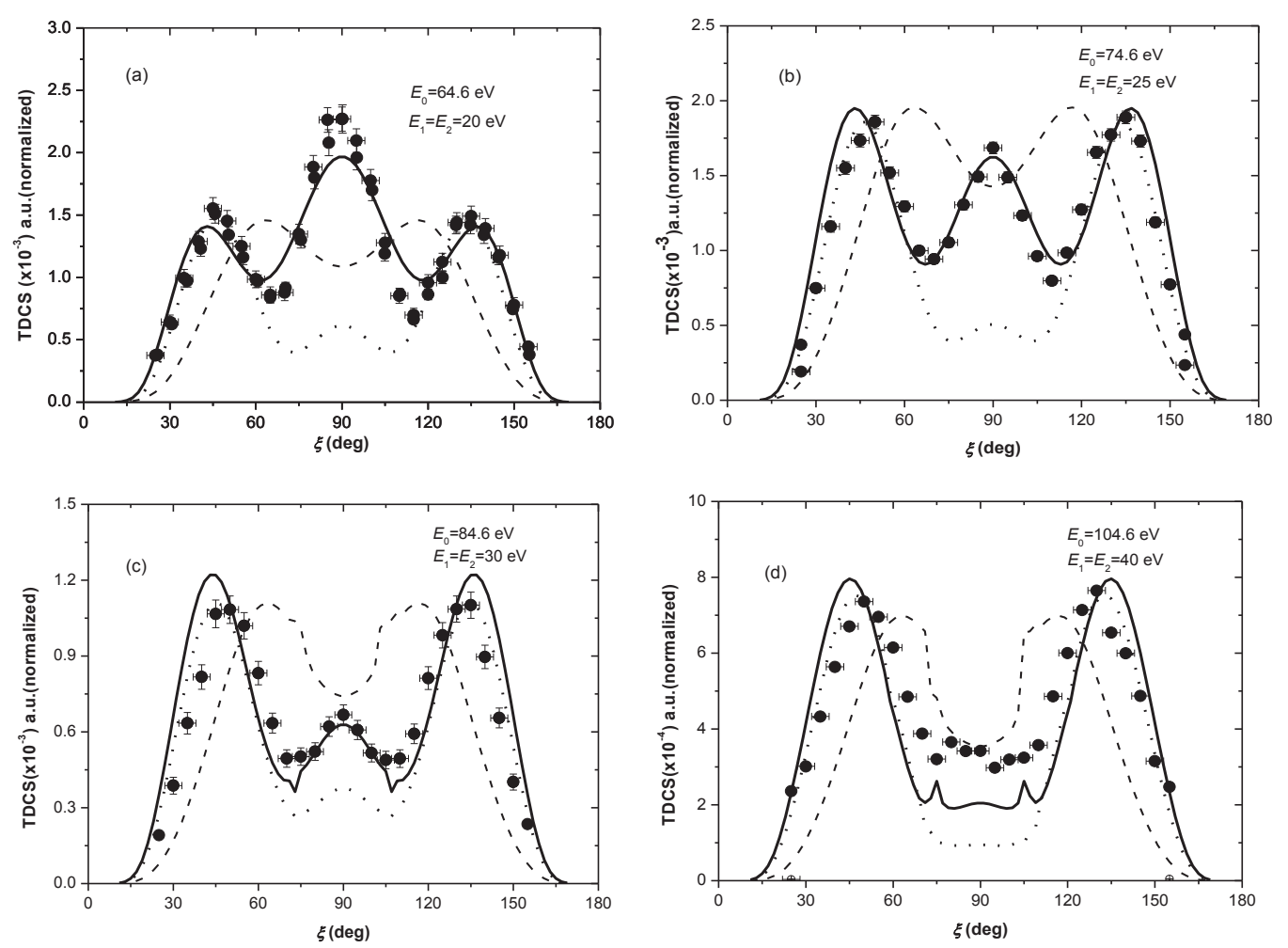

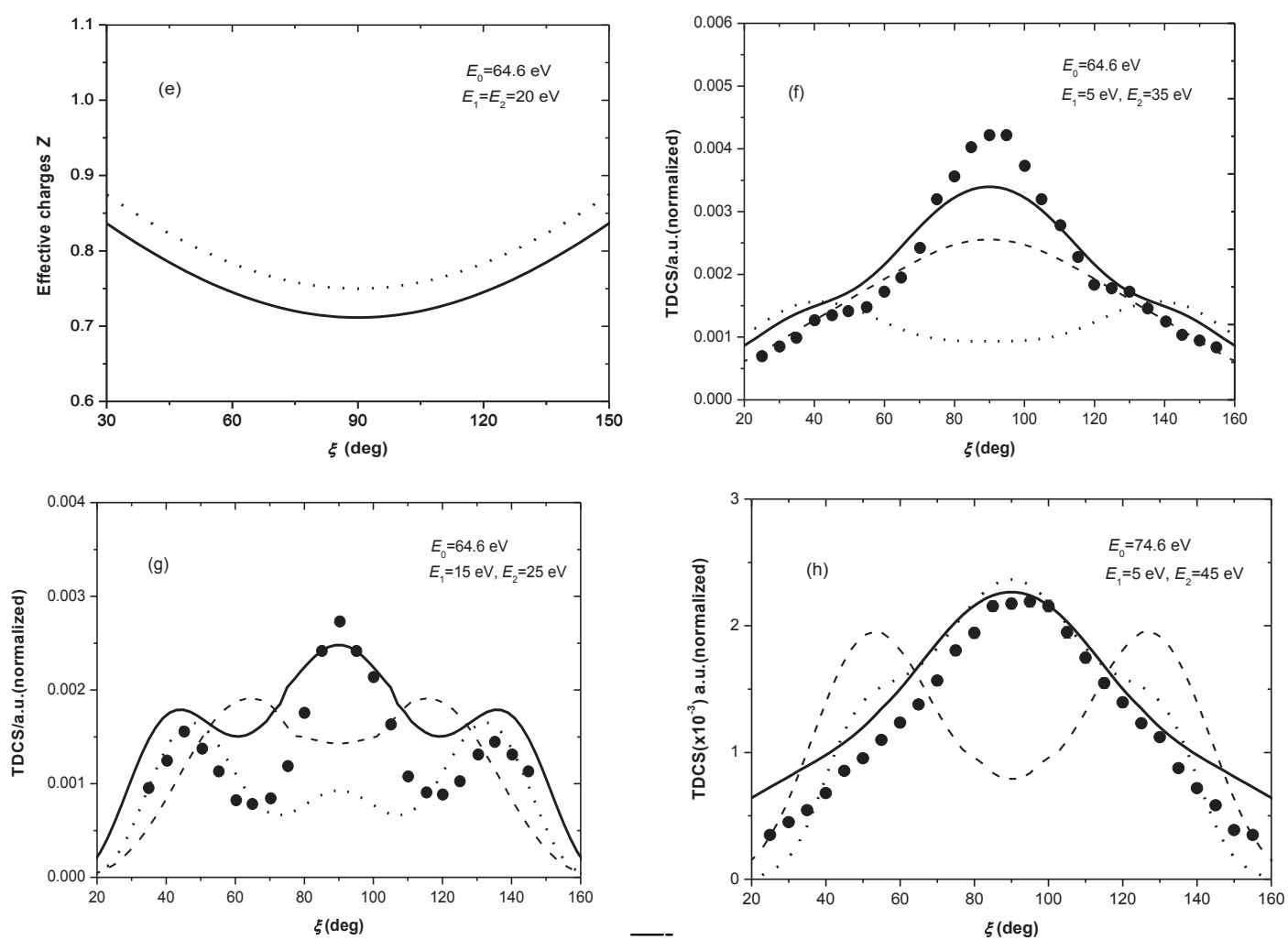

Figure 2: TDCS for ionizing helium. (a)-(d): in the perpendicular plane symmetric equal-energysharing geometry; $(f)-(h)$ : in the perpendicular plane symmetric unequal-energy-sharing geometry; (e): Effective charges $Z$ has been plotted as a function of the angle $\xi$ for Fig. 2(a). • experiment results $[15,17]$, BBK results, ...DS3C results, _this work.

body Coulomb interactions. It also description Strong shield effect and dynamical screening effects with shield of residual electron in the final state.

\section{Conclusion}

In conclusion, we apply BBK, DS3C model and the new modified Sommerfeld parameters of the DS3C model to calculation of TDCS for electron impact ionization of helium in the perpendicular plane symmetric geometry. The effective shield of residual electron is considered by the introduction of effective Sommerfeld parameters. And the new Sommerfeld parameters of the DS3C results show improvement over the BBK and DS3C model in obtaining better angular distribution and relative height of the present TDCS in comparison with experiment, which supports our conclusion that the peak structures of the present TDCS are to a large extent due to Strong DS effects of residual electron in the final state. It also shows the new Sommerfeld 
parameters to description of these effects are rather completely in the perpendicular plane symmetric geometry.

\section{References}

[1] M. Brauner, J. S. Briggs, and H. Klar, J. Phys. B: At. Mol. Opt. Phys. 22 (1989) 2265.

[2] J. Berakdar, Phys. Rev. A 53 (1996) 2314.

[3] S. Jones and D. H. Madison, Phys. Rev. A 62 (2000) 042701.

[4] K. Gao, H. Yang, X. J. Wu, and S. M. Zhang, J. At. Mol. Phys. 25 (2008) 683 (in Chinese).

[5] Y. Fang and K. Bartschat, J. Phys. B: At. Mol. Opt. Phys. 34 (2001) L19.

[6] S. Bellm, J. Lower, and K. Bartschat, Phys. Rev. Lett. 96 (2006) 223201.

[7] Z. Chen and D. H. Madison, J. Phys. B: At. Mol. Opt. Phys. 38 (2005) 4195.

[8] Z. J. Chen, D. H. Madison, and K. Bartschat, J. Phys.B: At. Mol. Opt. Phys. 40 (2007) 2333.

[9] S. M. Zhang, Z. J. Chen, and K. Xu, Acta Phys. Sin. 48 (1999) 453 (in Chinese).

[10] Z. J. Chen and K. Z. Xu, Chinese Phys. 10 (2001) 290.

[11] T. Rösel, C. Dupre, J. Röder, et al., J. Phys. B: At. Mol. Opt. Phys. 24 (1991) 3059.

[12] F. Mota-Furtado and P. F. O’Mahony, J. Phys. B: At. Mol. Opt. Phys. 22 (1989) 3925.

[13] J. Berakdar and J. S. Briggs, Phys. Rev. Lett. 72 (1994) 3799.

[14] A. J. Murray, F. H. Read, and N. J. Bowring, J. Phys. B: At. Mol. Opt. Phys. 30 (1997) 387.

[15] A. J. Murray, M. B. J. Woolf, and F. H. Read, J. Phys. B: At. Mol. Opt. Phys. 25 (1992) 3021.

[16] A. J. Murray and F. H. Read, J. Phys. B: At. Mol. Opt. Phys. 26 (1993) L359.

[17] K. L. Nixon, A. J. Murray, and C. Kaiser, J. Phys. B: At. Mol. Opt. Phys. 43 (2010) 085202. 